\title{
Conductas de higiene en el uso de estetoscopios de estudiantes de un programa de medicina de la ciudad de Tunja, Colombia
}

\author{
Hygiene behaviors in the use of stethoscopes
}

of students of a medicine program of the city of Tunja, Colombia

\begin{abstract}
Luis A. Torres-Ballesteros MD', Ana Y. Rodríguez-Sáenz MSc', Jenny A. Mendieta-Vergara MD'
\end{abstract}

Introducción: múltiples investigaciones han demostrado que los estetoscopios se encuentran colonizados por bacterias causantes de infecciones humanas. A pesar de ello, no se han definido las causas por las que estos instrumentos se contaminan. Objetivo: determinar las conductas de higiene que tienen los estudiantes de un programa de medicina de la ciudad de Tunja, Colombia, en el uso de los estetoscopios. Materiales y métodos: se seleccionaron 50 estudiantes de medicina quienes diligenciaron un cuestionario con preguntas cerradas, construido con base en grupos focales que permitieron caracterizar las conductas de uso intra y extrahospitalarias del estetoscopio. Además, a cada estudiante participante se le solicitó su estetoscopio para tomar muestras de cuatro partes del mismo, que posteriormente fueron cultivadas en agar nutritivo y caracterizadas macroscópica y microscópicamente por medio de la coloración de Gram. Resultados: de los 50 estudiantes que diligenciaron el cuestionario se evidenció que el 48\% nunca había limpiado el estetoscopio, el 24\% lo limpiaba con alcohol antiséptico y el $2 \%$ realizaba limpieza antes de la valoración del paciente. En el $100 \%$ de los estetoscopios se aislaron colonias bacterianas, principalmente cocos Gram positivos, siendo el diafragma la parte más contaminada de este instrumento. Conclusiones: se determinaron conductas inadecuadas en el uso del estetoscopio a nivel intra y extrahospitalario, así como la falta de información para su adecuada desinfección.

Palabras clave: higiene, conducta, estetoscopios, fómites, estudiantes, medicina.

Torres-Ballesteros LA, Rodríguez-Sáenz AY, Mendieta-Vergara JA. Conductas de higiene en el uso de estetoscopios de estudiantes de un programa de medicina de la ciudad de Tunja, Colombia. Medicina \& Laboratorio 2017; 23: 249-256.

${ }^{1}$ Estudiantes de Medicina, Universidad Pedagógica y Tecnológica de Colombia (UPTC). Tunja, Colombia. ${ }^{2}$ Bacterióloga, MSc. en Microbiología. Docente asociada del programa de Medicina, Facultad Ciencias de la Salud, Universidad Pedagógica y Tecnológica de Colombia (UPTC). Correspondencia: Calle 24 N. ${ }^{\circ}$ 5-63.

Correo electrónico: anayervid.rodriguez@uptc.edu.co

Conflicto de intereses: los autores declaran que no tienen conflicto de intereses Medicina \& Laboratorio 2017; 23: 249-256

Módulo 19 (Investigación), número 57. Editora Médica Colombiana S.A. 2017@ Recibido el 18 de mayo de 2017; aceptado el 09 de junio de 2017 
L as infecciones asociadas a la atención en salud, antes conocidas como nosocomiales o intrahospitalarias, son una de las principales preocupaciones que tienen los centros médicos al momento de prestar sus servicios, puesto que el padecimiento de alguna de estas por parte de los pacientes se traduce en un mayor tiempo de estancia hospitalaria y en el aumento de los recursos técnicos y profesionales, lo que incrementa los costos de hospitalización [1,2], sin contar el daño que pueda producir en los pacientes. Es bien conocido que estas infecciones pueden ser transmitidas a través de instrumentos de diagnóstico, evaluación y tratamiento, así como las manos e incluso la ropa de los trabajadores de la salud que tienen contacto con los pacientes, contaminados con microorganismos con potencial patogénico $[1,3,4]$. Por tal razón, en las instituciones de salud se han desarrollado guías de buena práctica que mitiguen este riesgo [5].

Desde hace varios años se han realizado investigaciones para identificar y aislar los microorganismos que se encuentran en cada uno de los instrumentos usados por el personal médico, y se ha confirmado que el estetoscopio se puede convertir en fómite [6-8] y que su uso sin adecuada profilaxis puede ser una de las fuentes de origen y transmisión de las infecciones asociadas a la atención en salud [3,9]. A pesar de que existen varios estudios que ratifican este hecho, e incluso muestran las estrategias para una adecuada limpieza que van desde el uso de alcohol etílico hasta de luz ultravioleta $[10,11]$, en la práctica aún persiste el rechazo por parte de los profesionales de la salud hacia el uso de desinfectantes o a la limpieza de este instrumento.

Al realizar la búsqueda de literatura en el tema, muy pocos estudios revelan los verdaderos motivos por los cuales se presenta esta situación, entre los cuales se encuentra la carencia de conocimiento del proceso de desinfección adecuado y la falta de ejemplo por parte de personas de mayor rango [12]. Por lo anterior, el presente estudio pretendía determinar las conductas de higiene que tienen los estudiantes de un programa de medicina, del sexto al décimo semestre, con sus estetoscopios, e identificar, macroscópica y microscópicamente, las bacterias contaminantes que se encuentran en ellos.

\section{Materiales y métodos}

\section{Población de estudio}

La población de estudio consistió en 50 estudiantes de un programa de medicina de la ciudad de Tunja, Colombia, que cumplieron con los siguientes criterios: portar estetoscopio propio, cursar del sexto al décimo semestre y participar voluntariamente en el estudio.

La investigación siguió las normas éticas para trabajos en la comunidad, tratadas en la resolución 008430 de 1993 del Ministerio de Salud de Colombia [13]. Previo al estudio se brindó la información del proyecto a los estudiantes, seguido de la firma del consentimiento informado.

\section{Recolección de información}

Las preguntas del cuestionario se formularon con base en los grupos focales de estudiantes que adelantaban la práctica clínica entre el sexto y décimo semestre. En estos grupos se trataron temas relacionados con las conductas de higiene en el uso del estetoscopio que portan los estudiantes durante su práctica hospitalaria; las reuniones fueron grabadas y analizadas posteriormente.

A partir de lo anterior, se construyó un cuestionario de doce preguntas de selección 
múltiple para caracterizar la limpieza del estetoscopio en términos de: frecuencia, tipo de desinfectante empleado, segmento limpiado, conductas de uso antes y después del contacto con el paciente, conocimiento de los métodos de desinfección del estetoscopio y su consideración como fómite; además de costumbres intra y extrahospitalarias para su uso, préstamo, transporte y custodia. Finalmente, se determinó la actitud frente a la desinfección de dicho instrumento.

\section{Muestras y recolección}

A los 50 estudiantes que participaron en el estudio se les solicitó el estetoscopio para tomar muestras de cuatro partes de estos (oliva derecha, oliva izquierda, manguera y diafragma) por medio de frotis con hisopo húmedo. Las muestras fueron sembradas en cajas de Petri con agar nutritivo, las cuales fueron divididas en cuatro partes rotuladas I: oliva derecha, II: oliva izquierda, III: manguera y IV: diafragma (véase figura $\mathbf{1}$ ).

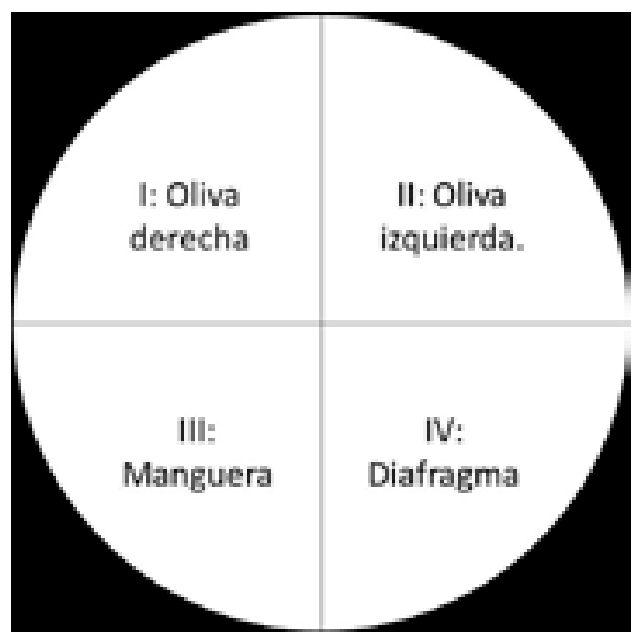

Figura 1. Disposición de las cajas de Petri para la siembra de las muestras tomadas a partir de los estetoscopios.
Las cajas de cultivo fueron llevadas a incubación por 24 horas a $37{ }^{\circ} \mathrm{C}$ y luego se analizaron macro y microscópicamente por medio de la coloración de Gram. Terminado el procesamiento de las muestras se socializaron los resultados con los participantes; además, se impartieron sesiones educativas sobre el buen uso del estetoscopio.

\section{Resultados}

\section{Distribución de la población de estudio}

En la investigación participaron 50 estudiantes, 30 del género masculino y 20 del femenino, la mayoría de ellos cursando el séptimo semestre del programa de medicina (véase figura 2 ).

\section{Conductas de higiene de los estetoscopios}

Con el cuestionario se evidenció que el $28 \%(14 / 50)$ de los estudiantes ha usado el estetoscopio por más de 24 meses desde su adquisición. Referente a la limpieza de este, el $48 \%$ (24/50) nunca la ha realizado, seguido por una frecuencia de limpieza entre 1 y 5 veces en el mes con un 44\% (22/50). Además, el 24\% (12/50) utilizaba como agente más común el alcohol antiséptico, seguido por un $16 \%$ (8/50) que empleaba toallas de papel con alcohol, mientras que el $50 \%(25 / 50)$ no utilizaba ninguna clase de antisépticos.

El $28 \%(14 / 50)$ de los estudiantes limpiaba específicamente el diafragma del estetoscopio y el $18 \%$ (9/50) lo limpiaba completamente. El 84\% (42/50) de los participantes se lavaba las manos antes de la valoración de los pacientes, convirtiéndose en la principal conducta de higiene, mientras que el 


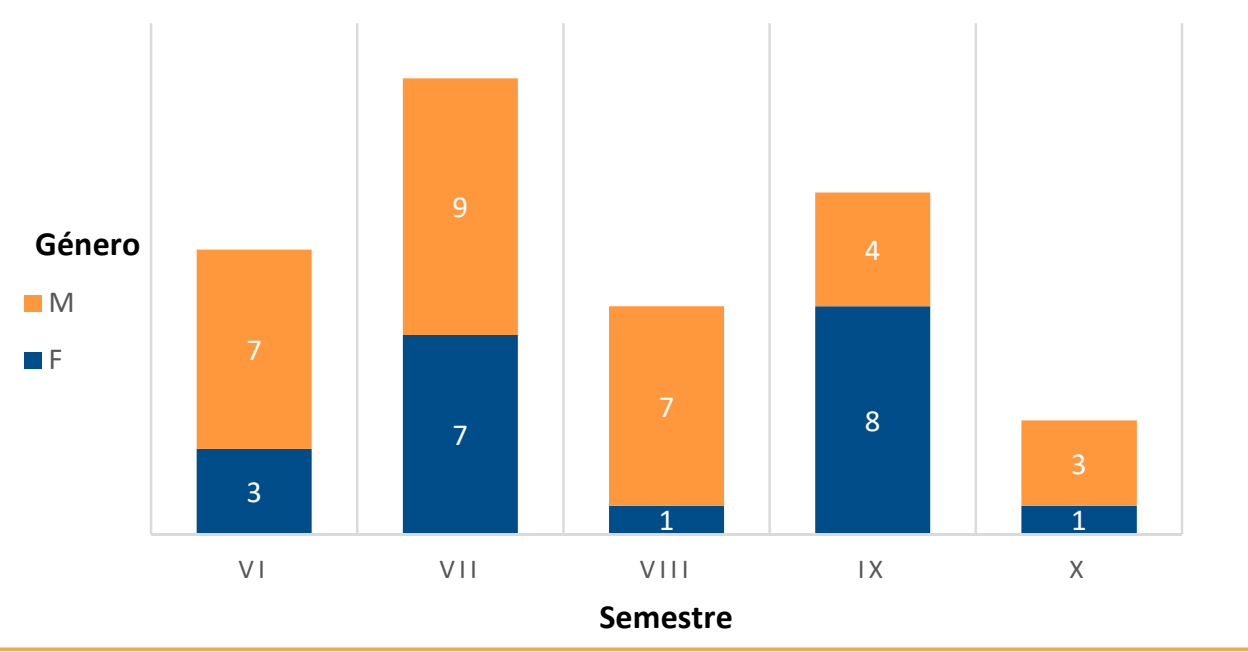

Figura 2. Caracterización de los participantes del estudio.

$2 \%(1 / 50)$ limpiaba el estetoscopio. También se estableció que el $86 \%$ (43/50) nunca había recibido instrucción de limpieza del estetoscopio.

En cuanto a las costumbres intrahospitalarias por parte de los estudiantes, el $64 \%$ $(32 / 50)$ transportaba el estetoscopio en el cuello y el $36 \%(18 / 50)$ en el bolsillo de la bata; el $72 \%$ (36/50) cuando no lo usan en el hospital lo guardan dentro de la maleta sin ser aislado de otros objetos. Una vez que los estudiantes finalizan la práctica clínica el $70 \%$ (35/50) lo lleva en la maleta, seguido del $16 \%$ (8/50) que lo envuelve en la bata. Finalmente, la disposición del estetoscopio en la vivienda es permanente en la maleta en el $54 \%(27 / 50)$, seguido por un $28 \%(14 / 50)$ que lo deja en su área de estudio (véase tabla $\mathbf{1}$ ).

\section{Estudio microbiológico}

De los 50 estetoscopios procesados para cultivo se aislaron colonias en el $100 \%$ de ellos. Del total de colonias aisladas el $34,2 \%$ provenían del cuadrante IV (diafragma), siendo este el más contaminado, seguido por los cuadrantes II (oliva izquierda) y III (manguera) con un $24,5 \%$ y $22,6 \%$, respectivamente, y por último, el cuadrante I (oliva derecha) con un $18,7 \%$ de las colonias.

En cuanto a la morfología bacteriana en el cuadrante I (oliva derecha) el 52\% correspondió a cocos Gram positivos, el $14 \%$ a bacilos Gram positivos, $14 \%$ bacilos Gram negativos y el 3,4\% a cocobacilos Gram negativos. En el cuadrante II (oliva izquierda) se evidenció un comportamiento similar con un $57,6 \%$ de cocos Gram positivos, $21 \%$ de bacilos Gram positivos, $11 \%$ de cocos Gram negativos y 7,9\% bacilos Gram negativos.

En el cuadrante III (manguera) la mayoría de las bacterias aisladas correspondieron a cocos Gram positivos en un $60 \%$, bacilos Gram positivos en un $17 \%$, cocobacilos Gram negativos en un $8,6 \%$ y cocos Gram negativos en un $5,7 \%$. Finalmente, en el cuadrante IV (diafragma) se encontró una morfología de cocos Gram positivos en el $57 \%$, bacilos Gram positivos en el $15 \%$, ba- 


\section{Tabla 1. Resultado cuestionario conductas de higiene de los estetoscopios ( $n=50)$}

\begin{tabular}{lll}
\hline Categoría & N. $^{\circ}$ & $\begin{array}{l}\text { Frecuencia } \\
\text { relativa (\%) }\end{array}$ \\
\hline $\begin{array}{l}\text { Tiempo de uso del estetoscopio } \\
\text { desde su adquisición (meses) }\end{array}$ & \\
$0-6$ & 9 & 18 \\
$7-12$ & 9 & 18 \\
$13-18$ & 8 & 16 \\
$19-24$ & 10 & 20 \\
Más de 24 & 14 & 28
\end{tabular}

Frecuencia de limpieza del estetoscopio (veces en el mes)

$\begin{array}{lll}\text { Nunca } & 24 & 48 \\ 1-5 & 22 & 44 \\ 6-10 & 2 & 4 \\ 11-15 & 2 & 4\end{array}$

Desinfectantes usados en la limpieza del estetoscopio

$\begin{array}{lll}\text { Alcohol antiséptico } & 12 & 24 \\ \text { Gel antibacterial } & 5 & 10 \\ \text { Toallas de papel con alcohol } & 8 & 16 \\ \text { Ninguno } & 25 & 50\end{array}$

Parte del estetoscopio que limpia

$\begin{array}{lll}\text { Embolo } & 2 & 4 \\ \text { Manguera } & 1 & 2 \\ \text { Diafragma } & 14 & 28 \\ \text { Todas } & 9 & 18 \\ \text { Ninguna } & 24 & 48\end{array}$

Práctica de higiene antes de valorar al paciente

\begin{tabular}{|c|c|c|}
\hline Lavado de manos & 42 & \\
\hline Limpieza de estetoscopio & 1 & \\
\hline Uso de guantes & 1 & \\
\hline Ninguna & 6 & \\
\hline $\begin{array}{l}\text { Instrucción de limpieza } p \\
\text { de la escuela o sitio de p }\end{array}$ & & \\
\hline No & 43 & \\
\hline Sí & 7 & \\
\hline
\end{tabular}

Transporte del estetoscopio durante la práctica Bolsillo de la bata $\quad 18 \quad 36$

$\begin{array}{lll}\text { Colgando en el cuello } & 32 & 64\end{array}$

Disposición del estetoscopio cuando no lo usa dentro del sitio de práctica

$\begin{array}{lll}\text { Bata } & 6 & 12 \\ \text { Bolsa } & 3 & 6 \\ \text { Colgando en el cuello } & 2 & 4 \\ \text { Casillero } & 3 & 6 \\ \text { Maleta } & 36 & 72\end{array}$

Disposición del estetoscopio después de la práctica

$\begin{array}{lll}\text { Maleta } & 35 & 70 \\ \text { Bolsa aislada de otros } & 3 & 6 \\ \text { objetos } & 8 & 16 \\ \text { Envuelto en la bata } & 1 & 2 \\ \text { Lo lleva en la mano } & 3 & 6 \\ \text { Guardado en un casillero } & & \end{array}$

Disposición de estetoscopio dentro de la vivienda

Dentro de la maleta $\quad 27 \quad 54$

Áreas comunes (comedor, $4 \quad 4$

sala) $\quad 4 \quad 8$

Armario con o sin ropa $\quad 14 \quad 28$

$\begin{array}{lll}\text { Área de estudio } & 1 & 2\end{array}$

No lo lleva

cilos Gram negativos en el 8\%, cocos Gram negativos y cocobacilos Gram positivos cada uno en un $4 \%$ y cocobacilos Gram negativos en el $2 \%$.

Cabe resaltar que en todos los cuadrantes también se identificaron levaduras entre un $2,6 \%$ y $17 \%$.

\section{Discusión}

El presente estudio determinó las conductas de higiene en el uso del estetoscopio de estudiantes de un programa de medicina antes, durante y después de la práctica clínica, donde se evidenció que el $86 \%$ (43/50) de los participantes no contaban con la información adecuada para la desinfección de este instrumento, debido, probablemente, a la ausencia de capacitación impartida por parte del centro educativo superior y el sitio de práctica, como se ha encontrado en otras investigaciones [14]. Otro aspecto importante a considerar es el personal clínico que labora en los hospitales, quienes no reconocen la importancia de desinfectar el estetoscopio, lo que lleva a que se omitan las indicaciones y recomendaciones de seguridad e higiene en los servicios de salud [6]. 
La carencia de información por parte de los estudiantes acerca de la limpieza de este instrumento ocasiona que un elevado porcentaje $(48 \%, 24 / 50)$ nunca lo desinfecte; hallazgo similar a lo encontrado en las investigaciones de Shiferaw y colaboradores (2013) [6] y Saunders y colaboradores (2013) [14] en las que el 98,1\% (102/104) y el $22,4 \%$ (69/308), respectivamente, de los estudiantes de medicina no realizaba la limpieza de su estetoscopio antes y después de examinar a cada paciente. Por otra parte, Uneke y colaboradores (2010) [7] también demostraron que el 35,6\% (26/73) de los médicos profesionales nunca habían lavado este instrumento y que el $100 \%$ de estos estaban colonizados por bacterias. Lo anterior hace pensar que los estudiantes, quienes no presentan unas buenas conductas de higiene en su formación, muy probablemente no las tendrán en su práctica profesional.

De igual manera, en el presente estudio las personas que consideraban que limpiaban su estetoscopio lo realizaban tan sólo 1 a 5 veces por mes, lo que en términos de frecuencia se considera irregular y es un comportamiento que se ha evidenciado en otras investigaciones $[15,16]$. Además, esta conducta es solo parcialmente cumplida de acuerdo con lo reglamentado por el Instituto Nacional de Salud de Colombia, quien recomienda que este tipo de instrumentos se debe limpiar y desinfectar diariamente antes de entrar en contacto con el paciente [17].

Diferentes autores han establecido que el alcohol etílico y el isopropílico son los desinfectantes de primera elección $[3,4,16]$. Jain y colaboradores (2013) [16] evaluaron la contaminación bacteriana en 80 estetoscopios y señalaron que solo el $27,5 \%$ de las personas a quienes pertenecían usaban alcohol para realizar la desinfección, porcentaje similar al encontrado en la presente investigación (24\%).

Por otra parte, entre los hábitos de higiene más comunes en el quehacer clínico se encuentra el lavado de manos, el cual se considera importante para evitar la transmisión de patógenos entre las personas [18]. En el presente estudio el $84 \%$ de la población realizaba el lavado de manos, lo cual es comparable con la investigación de Saunders y colaboradores (2013) [14] en la que el $92,2 \%$ (284/308) de los estudiantes reportaron que lo realizaban después de la atención de cada paciente. Esto representa un hallazgo significativo partiendo de que la ausencia de esta práctica se relaciona estrechamente con la contaminación del estetoscopio. En otro estudio se demostró que el porcentaje de los estetoscopios contaminados, y que pertenecían a los trabajadores de la salud con lavado de manos después de ver a cada paciente, fue del $28,5 \%$ (4/14); mientras que los del personal que no tenía dicha práctica de higiene lo estaban en un $86,0 \%(80 / 93)$ [7].

Los hallazgos de la morfología bacteriana del estudio determinaron que los estetoscopios se encontraron contaminados principalmente por cocos Gram positivos seguido por bacilos Gram negativos. Al revisar investigaciones como la de Bham y colaboradores (2015) [19] se detectó que la especie de cocos Gram positivos más prevalente como contaminante de los estetoscopios fue Staphylococcus aureus, como probablemente podría ocurrir en la presente investigación. Rao y colaboradores (2017) [20] encontraron que de un total de 52 estetoscopios analizados Staphylococcus aureus fue aislado en el $88,5 \%$ de los 
casos, todos ellos resistentes a la meticilina. Finalmente, en la revisión sistemática realizada por Haun y colaboradores (2016) [21] se evidencia que los estetoscopios, y otros instrumentos médicos, están comúnmente contaminados por patógenos Gram positivos como Staphylococcus aureus (incluyendo los resistentes a meticilina) y bacilos Gram negativos. Esto confirma la importancia que tienen las superficies inanimadas como reservorios, fómites y favorecedores de bacterias potencialmente patógenas que pueden llevar al desarrollo de infecciones asociadas a la atención en salud [22].

\section{Conclusiones}

Los estudiantes del programa de medicina presentan conductas de higiene inadecuadas en el uso del estetoscopio a nivel intra y extrahospitalario, por lo que es necesario desarrollar protocolos y sesiones educativas dirigidas a profesionales de la salud y estudiantes, en donde se informe sobre los métodos de desinfección más adecuados para los diferentes instrumentos de valoración del paciente y la transmisión de infecciones asociadas a la atención en salud. Gran parte de la literatura se centra en determinar cuáles son las actitudes hacia la limpieza del estetoscopio por parte de los estudiantes; sin embargo, no hay evidencia de los comportamientos dentro y fuera de los hospitales en cuanto a la disposición de este instrumento, lo cual fue determinado en este estudio.

\section{Agradecimientos}

A Nicolás Felipe Camargo Castillo y Anderson Fabián Calderón Cordero, estudiantes del programa de Medicina de la Universidad Pedagógica y Tecnológica de Colombia
(UPTC), por su colaboración en la recolección de las muestras y los datos.

\section{Bibliografía}

1. Schabrun S, Chipchase L. Healthcare equipment as a source of nosocomial infection: a systematic review. J Hosp Infect 2006; 63: 239-245.

2. Organización Mundial de la Salud. Una atención más limpia es una atención más segura. Ginebra, Suiza: OMS. 2017. Disponible: http://www.who.int/ gpsc/background/es/. Consultado: jun 2017.

3. Baptista-González HA, Zamorano-Jiménez CA. Estetoscopio, bata y corbata, y el riesgo de infecciones nosocomiales. Rev Invest Med Sur Mex 2011; 18 : 195-202.

4. Méndez Rodríguez IA, Calixto OJ, Becerra Cuervo WA, Vásquez JF, Bravo Ojeda JS, Pachón Barinas DP. Microorganismos presentes en fonendoscopios, manos, cavidad oral y nasal de estudiantes de una facultad de medicina. Rev Fac Med 2012; 20: 90100.

5. Otter JA, Yezli S, French GL. The role played by contaminated surfaces in the transmission of nosocomial pathogens. Infect Control Hosp Epidemiol 2011; 32: 687-699.

6. Shiferaw T, Beyene G, Kassa T, Sewunet T. Bacterial contamination, bacterial profile and antimicrobial susceptibility pattern of isolates from stethoscopes at Jimma University Specialized Hospital. Ann Clin Microbiol Antimicrob 2013; 12: 39.

7. Uneke CJ, Ogbonna A, Oyibo PG, Onu CM. Bacterial contamination of stethoscopes used by health workers: public health implications. J Infect Dev Ctries 2010; 4: 436-441.

8. Gopinath KG, Stanley S, Mathai E, Chandy GM. Pagers and stethoscopes as vehicles of potential nosocomial pathogens in a tertiary care hospital in a developing country. Trop Doct 2011; 41: 43-45.

9. Gupta N, Gandham N, Misra R, Jadhav S, Ujgare M, Vyawahare $\mathbf{C}$. The potential role of stethoscopes as a source of nosocomial infection. Med J DY Patil Univ 2014; 7: 156-159.

10. Messina G, Fattorini M, Nante N, Rosadini D, Serafini $\mathbf{A}$, Tani $\mathbf{M}$, et al. Time Effectiveness of Ultraviolet C Light (UVC) Emitted by Light Emitting Diodes (LEDs) in Reducing Stethoscope Contamination. Int J Environ Res Public Health 2016; 13.

11. Parmar RC, Valvi CC, Sira P, Kamat JR. A prospective, randomised, double-blind study of comparative efficacy of immediate versus daily cleaning of stethoscope using 66\% ethyl alcohol. Indian J Med 
Sci 2004; 58: 423-430.

12. Gazibara T, Radovanovic S, Maric G, Rancic B, Kisic-Tepavcevic D, Pekmezovic T. Stethoscope Hygiene: Practice and Attitude of Medical Students. Med Princ Pract 2015; 24: 509-514.

13. República de Colombia, Ministerio de Salud y Protección Social. Resolución número 8430 de 1993. 1993. Disponible: https://www.minsalud.gov.co/ sites/rid/Lists/BibliotecaDigital/RIDE/DE/DIJ/RESOLUCION-8430-DE-1993.PDF. Consultado: jun 2017.

14. Saunders C, Hryhorskyj L, Skinner J. Factors influencing stethoscope cleanliness among clinical medical students. J Hosp Infect 2013; 84: 242-244.

15. Singh DK, Cross DAR. Cross Sectional Study to Determine the Factors Associated with Stethoscope Hygiene Among the Health Care Providers in Tertiary Care Center. IJSR 2015; 4: 133-135.

16. Jain A, Shah H, Jain A, Sharma M. Disinfection of stethoscopes: Gap between knowledge and practice in an Indian tertiary care hospital. Ann Trop Med Public Health 2013; 6: 236-239.

17. República de Colombia, Ministerio de Salud y Protección Social, Instituto Nacional de Salud. Protocolo de Vigilancia en Salud Pública: Infecciones asociadas a dispositivos. 2016. Disponible: http://
www.ins.gov.co/lineas-de-accion/Subdireccion-Vigilancia/sivigila/Protocolos\%20SIVIGILA/PRO $\% 20$ Infecciones\%20asociadas\%20a\%20dispositivos.pdf. Consultado: jun 2017.

18. Scheithauer S, Haefner H, Schwanz T, Lopez-Gonzalez L, Bank C, Schulze-Robbecke R, et al. Hand hygiene in medical students: performance, education and knowledge. Int J Hyg Environ Health 2012; 215: 536-539.

19. Bham G, Bhandari J, Neupane MR, Dawadi R, Pradhan P. Aerobic Bacteria in the Diaphragmatic Portion of Stethoscope of Medical Professionals of Tertiary Care Hospital. JNMA J Nepal Med Assoc 2015; 53: 166-168.

20. Rao DA, Aman A, Muhammad Mubeen S, Shah A. Bacterial contamination and stethoscope disinfection practices: a cross-sectional survey of healthcare workers in Karachi, Pakistan. Trop Doct 2017; 47: 226-230.

21. Haun N, Hooper-Lane C, Safdar N. Healthcare Personnel Attire and Devices as Fomites: A Systematic Review. Infect Control Hosp Epidemiol 2016; 37: 1367-1373.

22. Maki DG. Stethoscopes and health care-associated infection. Mayo Clin Proc 2014; 89: 277-280.

Introduction: multiple investigations have shown that bacteria causing human infections colonize stethoscopes. However, the reasons why these instruments are contaminated have not been defined. Objective: To determine the hygiene behaviors that medicine students have in the use of stethoscopes. Materials and methods: 50 medicine students were selected to complete a questionnaire with closed questions, based on focus groups that allowed characterizing the intra and extrahospital behavior of the stethoscope use. In addition, each participant student was asked for his stethoscope in order to take samples of four parts of it, which were later culture on nutritive agar and characterized macroscopically and microscopically by Gram staining. Results: Of the 50 students, who completed the questionnaire, $48 \%$ have never cleaned the stethoscope, $24 \%$ cleaned it with antiseptic alcohol, and $2 \%$ cleaned before the patient's evaluation. In 100\% of the stethoscopes bacterial colonies were isolated, mainly Gram-positive cocci, been diaphragm the most contaminated part of this instrument. Conclusions: inadequate behaviors were determined in the intra- and extra-hospital stethoscope use, as well as lack of information for it adequate disinfection.

Key words: hygiene, behavior, stethoscopes, fomites, students, medicine. 